\title{
Multiple Concurrent IIInesses Associated with Anemia in HIV-Infected and HIV-Exposed Uninfected Children Aged 6-59 Months, Hospitalized in Mozambique
}

\author{
Caitlyn Duffy, ${ }^{1}$ Darlenne B. Kenga, ${ }^{2}$ Tebeb Gebretsadik, ${ }^{3}$ Fabião E. Maússe, ${ }^{2}$ Alice Manjate, ${ }^{2}$ Ernesto Zaqueu, ${ }^{4}$ \\ Hermenegilda F. Fernando, ${ }^{5}$ Ann F. Green, ${ }^{6}$ Jahit Sacarlal, ${ }^{2}$ and Troy D. Moon ${ }^{1,6 *}$ \\ ${ }^{1}$ Department of Pediatrics, Vanderbilt University Medical Center, Nashville, Tennessee; ${ }^{2}$ Department of Microbiology, Faculty of Medicine, \\ University Eduardo Mondlane, Maputo, Mozambique; ${ }^{3}$ Department of Biostatistics, Vanderbilt University Medical Center, Nashville, Tennessee; \\ ${ }^{4}$ Central Hospital Quelimane, Quelimane, Mozambique; ${ }^{5}$ General Hospital Mavalane, Maputo, Mozambique; ${ }^{6}$ Vanderbilt Institute for Global Health, \\ Vanderbilt University Medical Center, Nashville, Tennessee
}

\begin{abstract}
Anemia is an increasingly recognized problem in sub-Saharan Africa. To determine the magnitude, severity, and associated factors of anemia among hospitalized children aged 6-59 months, HIV-infected and HIV-exposed uninfected children (a child born to a known HIV-infected mother) with a documented fever or history of fever within the prior 24 hours of hospital admission $(N=413)$ were included in this analysis. Of 413 children enrolled, 364 (88\%) were anemic, with $53 \%$ classified as mild anemia (hemoglobin $[\mathrm{Hb}] 7-9.9 \mathrm{~g} / \mathrm{dL}$ ). The most common diagnoses associated with hospital admission included acute respiratory illness (51\%), malnutrition (47\%), gastroenteritis/diarrhea (25\%), malaria (17\%), and bacteremia (13\%). A diagnosis of malaria was associated with a decrease in $\mathrm{Hb}$ by $1.54 \mathrm{~g} / \mathrm{dL}(P<0.001)$. In HIV-infected patients, malaria was associated with a similar decrease in $\mathrm{Hb}(1.47 \mathrm{~g} / \mathrm{dL})$, whereas a dual diagnosis of bacteremia and malaria was associated with a decrease in $\mathrm{Hb}$ of $4.12 \mathrm{~g} / \mathrm{dL}(P<0.001)$. No difference was seen in $\mathrm{Hb}$ for patients on antiretroviral therapy versus those who were not. A diagnosis of bacteremia had a roughly 4 -fold increased relative odds of death during hospitalization (adjusted odds ratio $=3.97 ; 95 \% \mathrm{Cl}: 1.61,9.78 ; P=0.003$ ). The etiology of anemia in highburden malaria, HIV, tuberculosis, and poor nutrition countries is multifactorial, and multiple etiologies may be contributing to one's anemia at any given time. Algorithms used by physician and nonphysician clinicians in Mozambique should incorporate integrated and non-disease specific approaches to pediatric anemia management and should include improved access to blood culture.
\end{abstract}

\section{INTRODUCTION}

Children are the most vulnerable population to anemia worldwide. From 1993 to 2005, the global prevalence among preschool children was roughly $50 \%$, with approximately $30 \%$ of these coming from sub-Saharan Africa. ${ }^{1-5}$ Anemia in early childhood is a leading cause of hospital admission in subSaharan Africa and has been associated with reduced cognitive development and growth, and poor immune function, and is responsible for a high proportion of the malaria-related deaths each year. ${ }^{6-9}$ Public health efforts to target anemia have had limited success because of its multifactorial nature and have largely focused on populations considered to be at high risk, namely, pregnant women and children younger than 5 years in low-resource settings. ${ }^{10-12}$ Anemia in sub-Saharan Africa has historically been attributed to three principal causes: malaria, poor nutritional status with micronutrient deficiencies, and helminth infections, with HIV-associated anemia becoming a growing cause of total anemia burden due to opportunistic infections, adverse drug reactions, and untreated immunosuppression. ${ }^{13,14}$

In Mozambique, the contributors to anemia in hospitalized children aged less than 5 years largely mirror what is seen in other sub-Saharan Africa countries, with malnutrition, iron deficiency, malaria, and HIV being reported as the main associated factors. ${ }^{15}$ Mozambique is a country of approximately 29 million people as of 2017 and has one of the highest HIV prevalence rates in the region $\left(13.2 \%\right.$ in 2015). ${ }^{16,17}$ The number of persons on antiretroviral therapy (ART) in recent years has increased nearly 4-fold, from 309,000 in 2012 to 1.2

*Address correspondence to Troy D. Moon, Division of Pediatric Infectious Diseases, Department of Pediatrics, Vanderbilt Institute for Global Health, 2525 West End Ave., Suite 750, Nashville, TN. E-mail: troy.moon@vumc.org million in $2017 .^{18}$ Mozambique is further classified as one of the 30 high tuberculosis (TB) burden countries, in which it is estimated that nearly $60 \%$ of TB patients are coinfected with HIV. $^{19}$ Malnutrition remains a significant problem in the country, with stunting prevalence one of the highest in Africa, roughly $44 \%$ in $2015 .{ }^{20}$ Malaria accounted for approximately $30 \%$ of all deaths in 2017 , with the bulk of this mortality occurring in children aged less than 5 years. ${ }^{21,22}$ Finally, a study of all children aged less than 5 years hospitalized in Maputo in February and March 2009 showed a prevalence of pathogenic intestinal parasites at $16.1 \% .^{23}$

In recent years, the burden of anemia has been garnering increasing attention; although targeted, context-specific knowledge of its epidemiology and severity across geographic areas remains limited. ${ }^{11,15}$ Because nationally representative data on anemia are frequently insufficient or unavailable, we often estimate its prevalence based on available subpopulation data and then extrapolate to estimate the overall population-level prevalence. ${ }^{1}$ Despite increased attention in the scientific community at large, at the bedside in sub-Saharan Africa, anemia frequently does not get the attention it deserves, likely resulting from clinicians viewing it as a consequence of other disease processes. ${ }^{15}$ This is highlighted by one report, for example, in which adherence to international guidelines for transfusion was poor in the case of severe anemia, and most patients requiring blood did not get it. ${ }^{24}$ As a result, anemia is likely a much more widespread and under-recognized public health problem in low-resource countries, not only contributing to poor health outcomes in children but also limiting their future socioeconomic potential due to impaired cognitive development. ${ }^{6,15,25}$

Our objective was to describe the magnitude, severity, and associated factors of anemia among HIV-infected and HIV-exposed uninfected hospitalized children aged 6-59 
months in two regionally distinct areas of Mozambique. Identifying the magnitude of anemia and its associated factors in this high-risk population could inform future interventions and health practices that aim to reduce the prevalence of anemia in Mozambique.

\section{MATERIALS AND METHODS}

Study design and population. We conducted a prospective observational study of HIV-infected and HIVexposed uninfected children in the cities of Maputo and Quelimane, Mozambique, who were prospectively followed up during their hospital stay. This "parent" study was designed to determine the incidence, etiology, antibiotic sensitivity patterns, and molecular characterizations of culture-confirmed bacteremia in representative rural and urban hospitals in Mozambique. All HIV-infected and HIVexposed uninfected children aged 0-59 months with a documented axillary temperature of $\geq 37.5^{\circ} \mathrm{C}$ or rectal temperature $\geq 38.0^{\circ} \mathrm{C}$, or a history of fever within the 24 hours before hospitalization between April 1, 2016 and December 31, 2018 were enrolled. Patients were recruited from the pediatric urgent care clinics of three hospitals in Maputo: Central Hospital Maputo, Hospital Jose Macamo, and General Hospital Mavalane; and two hospitals in Quelimane: Central Hospital Quelimane and General Hospital Quelimane. All are tertiary referral hospitals supported by the National Health System (Figure 1).

Using data collected during the course of the parent study, we conducted this anemia analysis on our patient population aged between 6 and 59 months.

Definitions. Children were considered HIV positive if they had a documented proof of HIV infection by polymerase chain reaction (PCR) or HIV rapid antibody tests (if $\geq 9$ months at time of test), or if they were taking ART in the absence of documented HIV test results. Children admitted with a fever,

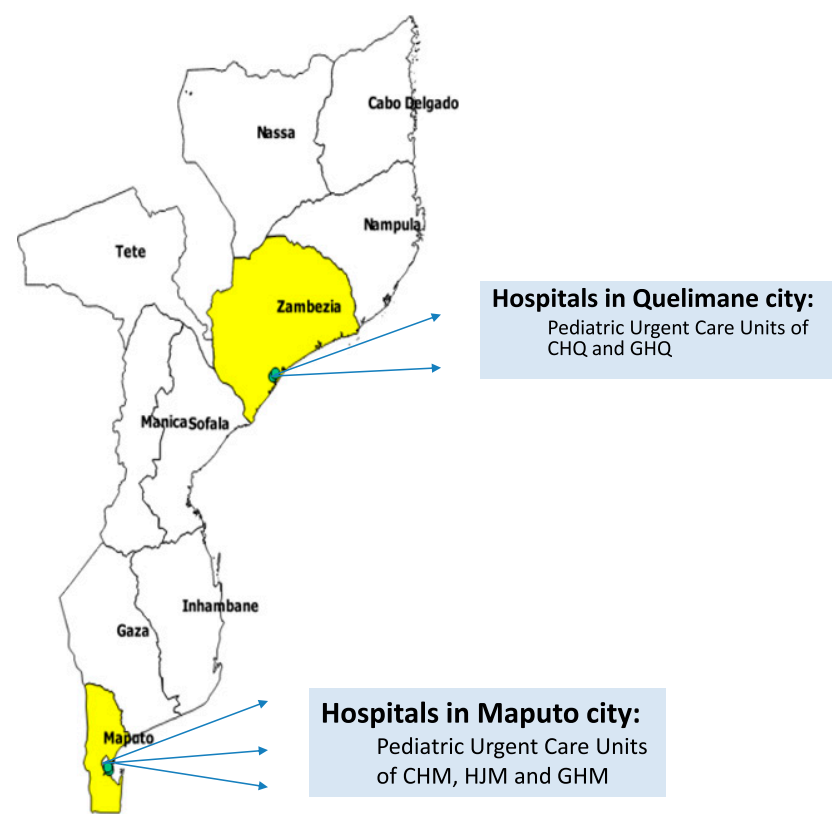

FIGURE 1. Map of Mozambique with study hospitals identified for Maputo and Quelimane. This figure appears in color at www.ajtmh.org. or a history of fever, and no documented history of HIV, but with documented maternal HIV exposure by self-report, were offered a PCR (if $<9$ months old) or an HIV rapid antibody test (if $\geq 9$ months old). Those with negative test results were then considered HIV-exposed uninfected. Children admitted with a fever, or a history of fever, and no documented history of HIV and no documented maternal HIV exposure by selfreport, although with high clinical suspicion of HIV, were enrolled and offered HIV testing based on the aforementioned. Those with negative test results were considered HIV negative, and there were 18 subjects who were excluded from analysis because of a final designation of being HIV negative or unknown.

For all eligible patients, study staff collected a single blood specimen for bacterial culture before the initiation of antibiotics. Additional diagnostic testing was performed if there was clinical suspicion based on the history or signs/symptoms. Readily available tests included complete blood count, blood chemistries, dipstick urinalysis, chest X-ray, lumbar puncture for chemistries and bacterial culture, stool culture, stool ova and parasites, HIV rapid antibody testing or DNA-PCR, and malaria antigen rapid testing. For patients with a positive malaria rapid test, thick and thin blood films were prepared for confirmation and to quantify Plasmodium falciparum parasitemia. There was no available laboratory diagnostics for TB in this age-group.

Severe anemia was defined as a hemoglobin $(\mathrm{Hb})$ concentration of $<7 \mathrm{~g} / \mathrm{dL}$, moderate anemia as $\mathrm{Hb}$ of $7-9.9 \mathrm{~g} / \mathrm{dL}$, mild anemia as $\mathrm{Hb}$ of $10-10.9 \mathrm{~g} / \mathrm{dL}$, and no anemia if $\mathrm{Hb}$ was $>11$ $\mathrm{g} / \mathrm{dL}$. All patients were followed up until their discharge from the hospital. Possible final disposition included discharged patients, patients who died during hospitalization, or patients who abandoned treatment, meaning they left the hospital against the wishes of the treating clinician.

Data collection. Data for the parent study, including this anemia analysis, were collected by study clinicians using a paper-based study instrument and then uploaded into a password protected, tablet-based, online database maintained by the Research Electronic Data Capture consortium (www.project-redcap.org). This allowed for the recording of demographic information, medical and medication history, and information on clinical course while hospitalized. Data quality control was conducted by study investigators who reviewed all completed paper-based study instruments and confirmed the accuracy of data entered into the electronic database.

Statistical analysis. Descriptive statistics were used to summarize the participants' sociodemographic characteristics using frequencies and proportions (for dichotomous or categorical variables) or medians with interquartile ranges for continuous variables. In univariate analysis, we compared factors by anemia status defined as $>11 \mathrm{~g} / \mathrm{dL}, 10-10.9 \mathrm{~g} / \mathrm{dL}$, $7-9.9 \mathrm{~g} / \mathrm{dL}$, and $<7 \mathrm{~g} / \mathrm{dL}$ using chi-squared test. In regression analysis, for our response or outcome variable that proxies anemia, we used $\mathrm{Hb}$ level $(\mathrm{g} / \mathrm{dL}$ ) without categorization. Using the continuous response of the $\mathrm{Hb}$ level maximizes our regression power by using all information levels. We assessed the association of prespecified diagnosed conditions of interest that included bacteremia, malaria, gastroenteritis (GE)/ diarrhea, acute respiratory illness (ARI), or malnutrition, with the $\mathrm{Hb}$ level as a biomarker response variable using separate regression analyses. Because anemia can be secondary to 
one's ART treatment, we also examined the association of ART treatment and $\mathrm{Hb}$ level among HIV-infected patients. We examined the interaction effect of concurrent diagnoses using separate regression and a cross-product term (bacteremia and malaria, bacteremia and malnutrition, and malaria and malnutrition) on $\mathrm{Hb}$ levels as an exploration analysis. Among our subset of children who were HIV infected, we examined the association of diagnosis with the outcome of in-hospitalization death (yes versus no) using multivariable logistic regression. Each multivariable regression analysis included the child's age, gender, and health facility as covariates for adjustment.

We had case-wise deletions on $\sim 4 \%$ of subjects because of missing data for infant age $(n=18)$. We performed multiple imputations (Mls) to account for missing age data in multivariable regression as sensitivity analyses. ${ }^{26}$ We present beta coefficient-associating factors with anemia from complete case analysis. Statistical significance was determined using a 2-sided, 5\% significance level. Statistical analysis was performed using R version 3.4.0 software (R core team, 2015, R Foundations for Statistical Computing, Viena, Austria, http:// www.r-project.org).

Ethical considerations. The Mozambican National Bioethics Committee for Health (Comité Nacional de Bioética para Saúde) (404/CNBS/14) and the Institutional Review Board of Vanderbilt University Medical Center (IRB\#141167) approved this analysis. Informed consent was obtained from the parent or legal guardian of all children enrolled in this study.

\section{RESULTS}

Patient characteristics. A total of 413 HIV-infected (95\%) and HIV-exposed uninfected (5\%) children aged 6-59 months were enrolled in our study between April 1, 2016 and December 31,2018 , of which $57 \%$ were male and $36 \%$ were aged 6-12 months (Table 1). Two hundred twenty-three children (54\%) were recruited from hospitals in Quelimane, and 190 children (46\%) from hospitals in Maputo (Figure 1). Three hundred sixty-four (88\%) children were anemic at admission, defined by $\mathrm{Hb}$ of $\leq 11.0 \mathrm{~g} / \mathrm{dL}$. Most $(53 \%)$ children were classified as having moderate anemia ( $\mathrm{Hb} 7-9.9 \mathrm{~g} / \mathrm{dL})$, and the median $\mathrm{Hb}$ level was $8.7 \mathrm{~g} / \mathrm{dL}$ (IQR: 7.3, 10.0). The five most common diagnoses associated with hospital admission included ARI (51\%), malnutrition (47\%), GE/ diarrhea (25\%), malaria (17\%), and bacteremia (13\%). Overall, 40 children (10\%) in the cohort died during hospitalization.

Anemia status and diagnosis. We classified patients by their anemia status as well as the five most common hospital diagnoses encountered (Table 2). Across all diagnoses, the largest proportion of children (between 34\% and 59\%) was classified as having moderate anemia. Those with a malaria diagnosis had a higher proportion of cases classified as severe anemia (46\%, $P<0.001)$, compared with patients without a malaria diagnosis. When we compared distribution of $\mathrm{Hb}$ levels by diagnosis, we observed statistically significant lower $\mathrm{Hb}$ levels for children diagnosed with malaria (median 7.4 IQR: 5.7-9.0 versus median 8.8 IQR: 7.7-10.1; $P<0.0001$ ) and a statistically significant increased $\mathrm{Hb}$ level for children diagnosed with GE/diarrhea $(P<0.0001)$ (Figure 2).

We then examined anemia status in children receiving ART. Overall, the largest proportion of children was classified as
TABLE 1

Sociodemographics of children aged 6-59 months hospitalized in Maputo and Quelimane, April 2016-December 2018

\begin{tabular}{|c|c|}
\hline$N=413$ & Total, $N(\%)$ \\
\hline \multicolumn{2}{|l|}{ Gender } \\
\hline Male & $236(57)$ \\
\hline Female & $177(43)$ \\
\hline \multicolumn{2}{|l|}{ Age (months) } \\
\hline $6-12$ & $148(36)$ \\
\hline $13-24$ & $151(37)$ \\
\hline $25-59$ & $96(23)$ \\
\hline Missing data & $18(4)$ \\
\hline \multicolumn{2}{|l|}{ Hospital } \\
\hline General Hospital Quelimane & $128(31)$ \\
\hline Central Hospital Quelimane & $95(23)$ \\
\hline General Hospital Mavalane (Maputo) & $106(26)$ \\
\hline Hospital Jose Macamo (Maputo) & $71(17)$ \\
\hline Central Hospital Maputo & $13(3)$ \\
\hline Hb level (g/dL), median [IQR] & $8.7[7.3,10.0]$ \\
\hline \multicolumn{2}{|l|}{ Anemia status } \\
\hline Non-anemic (Hb > $11 \mathrm{~g} / \mathrm{dL})$ & $49(12)$ \\
\hline Mild anemia (Hb 10-10.9 g/dL) & 59 (14) \\
\hline Moderate anemia ( $\mathrm{Hb} 7-9.9 \mathrm{~g} / \mathrm{dL})$ & 217 (53) \\
\hline Severe anemia $(\mathrm{Hb}<7 \mathrm{~g} / \mathrm{dL})$ & $88(21)$ \\
\hline \multicolumn{2}{|l|}{ HIV status $\ddagger$} \\
\hline Positive & $393(95)$ \\
\hline HIV-exposed uninfected & $20(5)$ \\
\hline On $\mathrm{ART}^{\star}(n=408)$ & $231(57)$ \\
\hline \multicolumn{2}{|l|}{ Hospital diagnosis $\ddagger$} \\
\hline ARI & $210(51)$ \\
\hline Malnutrition & $196(47)$ \\
\hline Gastroenteritis/diarrhea & $105(25)$ \\
\hline Malariat & $71(17)$ \\
\hline Bacteremia & $54(13)$ \\
\hline Tuberculosis & $25(6)$ \\
\hline Helminth infection & $5(1)$ \\
\hline Dermatitis & $5(1)$ \\
\hline Seizures & $9(2)$ \\
\hline Other & $93(23)$ \\
\hline Death during hospitalization & $40(10)$ \\
\hline
\end{tabular}

moderate anemia (51\%) (Table 2). No statistically significant difference was detected in the $\mathrm{Hb}$ level for patients on ART versus those who were not (Figure 2).

Among both HIV-infected and HIV-exposed uninfected children using separate multivariable linear regression analyses that assessed the association of hospital diagnosis with the $\mathrm{Hb}$ level, a diagnosis of malaria was associated with an average decrease in $\mathrm{Hb}$ by $1.54 \mathrm{~g} / \mathrm{dL}(P<0.001)$ versus those who did not have malaria. A diagnosis of GE/diarrhea was associated with an average increase in $\mathrm{Hb}$ of $0.92 \mathrm{~g} / \mathrm{dL}(P<$ 0.001) (Table 3). When analyzed among HIV-infected children only, similar trends were seen for both malaria and GE/ diarrhea.

A large proportion of our HIV-infected population presented with multiple concurrent illnesses at the time of hospitalization. When we explored the interaction effect of these concurrent illnesses on $\mathrm{Hb}$ levels, we found that in HIV-infected patients admitted with both a bacteremia and a malaria diagnosis concurrently, there was an average decrease in $\mathrm{Hb}$ of $4.12 \mathrm{~g} / \mathrm{dL}$ compared with children without bacteremia or malaria $(P<0.001)$. We additionally looked at HIV-infected patients with a dual diagnosis of malaria and malnutrition (average $\mathrm{Hb}$ decrease of $1.28 \mathrm{~g} / \mathrm{dL}, P<0.0001$ ) and dual 
TABLE 2

Anemia status (g/dL) of children aged 6-59 months hospitalized in Maputo and Quelimane, Mozambique

\begin{tabular}{|c|c|c|c|c|c|}
\hline \multirow[b]{2}{*}{$N=413$} & \multicolumn{5}{|c|}{ Anemia (g/dL) } \\
\hline & Non-anemic $(\mathrm{Hb}>11), N(\%)$ & Mild (Hb 10-10.9), N (\%) & Moderate (Hb 7-9.9), $N(\%)$ & Severe $(\mathrm{Hb}<7), N(\%)$ & $P$-value ${ }^{*}$ \\
\hline \multicolumn{6}{|l|}{ Hospital diagnosis } \\
\hline Bacteremia $(n=54) \dagger$ & $8(15)$ & $8(15)$ & $217(53)$ & $88(21)$ & 0.25 \\
\hline Malaria $(n=71) \dagger$ & $8(11)$ & $6(8)$ & $24(34)$ & $33(46)$ & $<0.001$ \\
\hline Malnutrition $(n=196)$ & $20(10)$ & 24 (12) & $110(56)$ & $42(21)$ & 0.41 \\
\hline ARI $(n=210)$ & $21(10)$ & $35(17)$ & $113(54)$ & $41(20)$ & 0.29 \\
\hline GE/diarrhea $(n=105)$ & $18(17)$ & $16(15)$ & $62(59)$ & $9(9)$ & 0.002 \\
\hline On ART $(n=231) \ddagger$ & $23(10)$ & 33 (14) & $117(51)$ & $58(25)$ & 0.22 \\
\hline Death during hospitalization $(n=40)$ & $2(5)$ & $2(5)$ & $31(78)$ & $5(12)$ & 0.01 \\
\hline
\end{tabular}

diagnosis of bacteremia and malnutrition (average $\mathrm{Hb}$ decrease of $0.67 \mathrm{~g} / \mathrm{dL}, P=0.156$ ). Although both saw relative decreases in $\mathrm{Hb}$, the decreases were of similar magnitude to malaria alone or bacteremia alone (Table 4).
Hospital mortality. In multiple logistic regression, HIVinfected patients who presented to the hospital with bacteremia had a roughly 4-fold increased relative odds of dying during their hospitalization $(P=0.003)$. Those with $A R I$ had a
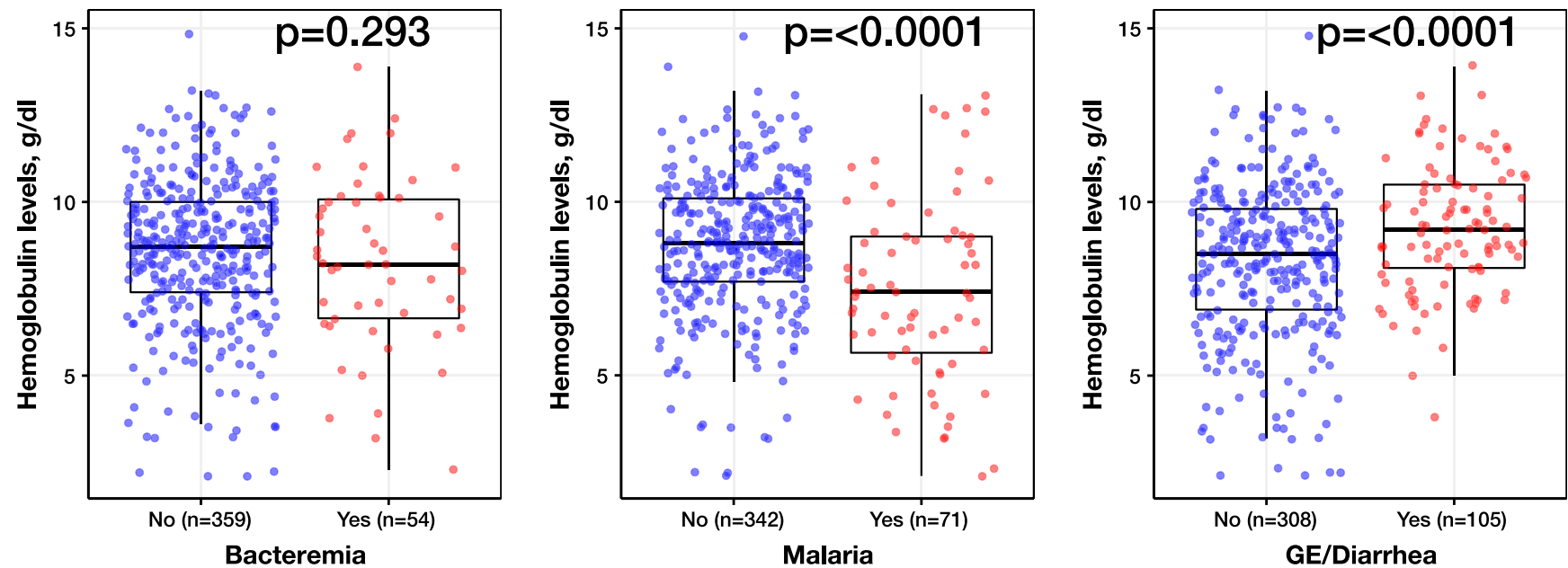

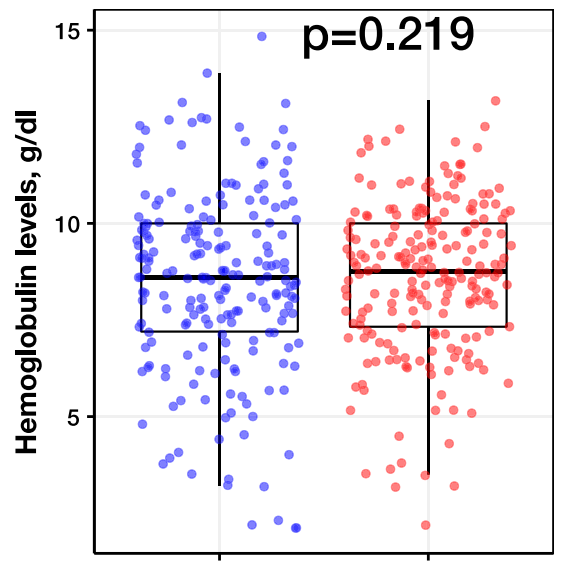

No $(n=203) \quad$ Yes $(n=210)$

Acute respiratory illness

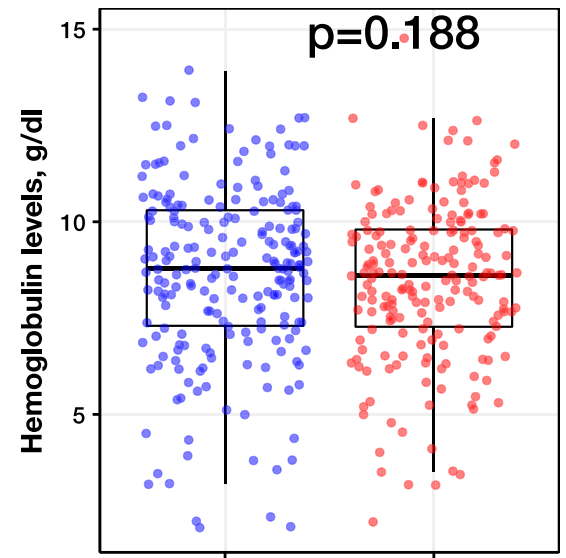

No $(n=217) \quad$ Yes $(n=196)$

Malnutrition

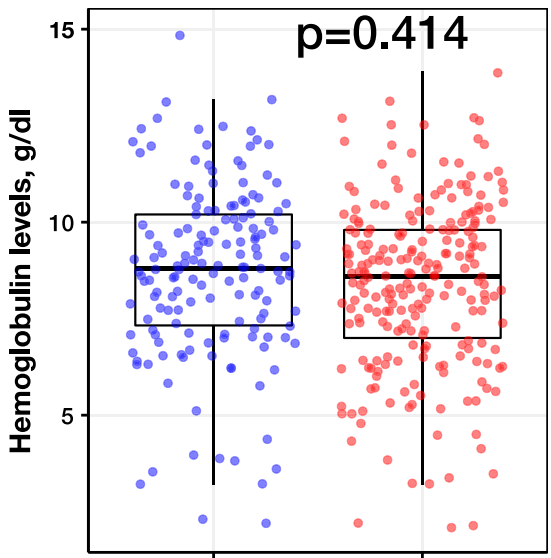

No $(n=158) \quad$ Yes $(n=231)$

ART status among HIV positive only

FIGURE 2. Box plots of hemoglobin level $(\mathrm{g} / \mathrm{dL})$ at the time of hospitalization ( $y$ axis) by the diagnosed conditions of interest ( $x$ axis). The median (middle bar), first quartile (lower bar), and third quartile (upper bar) are shown. Scatter plot values of each patient's hemoglobin level was overlaid by diagnosed conditions. The sample size of each group is also shown. $P$-values were obtained from separate multivariable linear regressions that adjusted for child's age, gender, and health facility/center. *Antiretroviral therapy (ART) status = on ART. This figure appears in color at www.ajtmh.org. 
TABLE 3

Association of hospital diagnosis and $\mathrm{Hb}$ level $(\mathrm{g} / \mathrm{dL})$ among HIV-infected and HIV-exposed uninfected children

\begin{tabular}{|c|c|c|c|c|c|c|}
\hline & \multicolumn{4}{|c|}{ HIV-infected and HIV-exposed uninfected children } & \multicolumn{2}{|c|}{ HIV-infected only } \\
\hline & Unadjusted $\beta(95 \% \mathrm{Cl})$ & $P$-value & Adjusted $^{\star} \beta(95 \% \mathrm{Cl})$ & $P$-value & Adjusted $^{*} \beta(95 \% \mathrm{Cl})$ & $P$-value \\
\hline Bacteremia $†$ & $-0.32(-0.95,0.30)$ & 0.313 & $-0.34(-0.97,0.29)$ & 0.293 & $-0.57(-1.23,0.09)$ & 0.092 \\
\hline Malaria† & $-1.31(-1.86,-0.77)$ & $<0.001$ & $-1.54(-2.11,-0.96)$ & $<0.001$ & $-1.47(-2.06,-0.88)$ & $<0.001$ \\
\hline Malnutrition & $-0.21(-0.64,0.21)$ & 0.322 & $-0.31(-0.78,0.15)$ & 0.188 & $-0.35(-0.83,0.14)$ & 0.164 \\
\hline Acute respiratory illness & $0.11(-0.31,0.53)$ & 0.618 & $0.28(-0.16,0.73)$ & 0.219 & $0.24(-0.22,0.70)$ & 0.297 \\
\hline Gastroenteritis/diarrhea & $0.90(0.42,1.37)$ & $<0.001$ & $0.92(0.42,1.42)$ & $<0.001$ & $0.92(0.40,1.44)$ & $<0.001$ \\
\hline
\end{tabular}

$\mathrm{Hb}=$ hemoglobin.
${ }^{*}$ Multiple linear regression models for hospital diagnosis and $\mathrm{Hb}$ levels as a biomarker for anemia.

† Laboratory-confirmed diagnosis.

slightly higher relative odds of death during their hospitalization (adjusted odds ratio [aOR]: $1.18 ; 95 \% \mathrm{Cl}: 0.29-2.06 ; P=$ 0.009 ) (Table 5). There was no statistically significant association with in-hospital death in patients presenting with malaria (aOR: $0.55 ; 95 \% \mathrm{Cl}: 0.14,2.07 ; P=0.373$ ).

\section{DISCUSSION}

The majority of patients (88\%) in our cohort of children aged 6-59 months were anemic on admission, with 53\% being moderately anemic and $21 \%$ being severely anemic. This is much higher than the global prevalence of anemia ( 50\%) reported for similarly aged children and slightly higher than what has been reported elsewhere in sub-Saharan Africa (60-70\%), underscoring the importance of anemia as a severe public health threat in this region of the world. . $^{1,2,9,15,25}$

According to Mozambique's latest national demographic health survey conducted in 2015, the prevalence of anemia in children aged 6-59 months was 64\%, with dramatic differences reported between the two provinces represented in this study, $77 \%$ for Zambézia Province and $45 \%$ for Maputo City. ${ }^{17}$ Although we saw no statistical differences in the prevalence of anemia by geographic region in our hospital-based cohort, the high overall prevalence of anemia seen in our study and the lack of geographic difference could be due to several factors. First, our cohort is made up of children hospitalized with an acute febrile illness and undoubtedly represents a group with a higher frequency of comorbidities. Second, our study focused on HIVinfected and/or HIV-exposed uninfected children for analysis. HIV itself can inherently cause anemia because of depressed hematopoiesis. ${ }^{27}$ In addition, for patients who have initiated ART and prophylaxis for opportunistic infections with drugs such as sulfamethoxazole/trimethoprim (cotrimoxazole), there is a risk of developing drug-induced anemia. In Ethiopia, for example, in pediatric patients who had initiated ART, the type of regimen and the duration on ART were predictors found to be associated with anemia. ${ }^{28}$ However, the association found between anemia and cotrimoxazole prophylaxis has been mixed. Cotrimoxazole may directly impact erythropoiesis, causing a reduction in red cell number, yet simultaneously lead to increased $\mathrm{Hb}$ levels because of its beneficial effects at reducing malaria and other infections. ${ }^{29-31}$ Even still, our study results suggest a cause for concern, as HIV-associated anemia has also been shown to increase the risk of mortality. ${ }^{13}$

Concern about the high prevalence of anemia in our cohort is heightened by the fact that many HIV-infected and HIVexposed uninfected children in Mozambique are at increased risk for other processes which affect the production of red blood cells, such as nutritional deficiencies from chronic malnutrition, chronic blood loss from helminth infections, and endemic infections such as malaria and TB. ${ }^{13,32-34}$ Moreover, children hospitalized in Mozambique frequently have multiple concurrent illnesses that can simultaneously contribute to anemia. We found that children aged 6-59 months who were diagnosed with malaria had statistically significant decreases in $\mathrm{Hb}$ levels, with differences in the average $\mathrm{Hb}$ level that appears enhanced in those concomitantly diagnosed with both malaria and bacteremia. In addition, HIV-infected children diagnosed with either bacteremia or ARI were at increased odds of death during their hospitalization.

Therefore, anemia observed in regions with a high burden of HIV infection requires more attention and is likely more multifactorial than that observed in HIV-negative populations. ${ }^{35}$ In Mozambique, specific guidelines have been created for the management and treatment of HIV-infected ambulatory adults with anemia to steer clinician approaches away from empiric treatment and more toward an evidence-based approach to diagnosis and treatment. However, similar tools are less specific for hospitalized patients and for pediatric populations. ${ }^{13}$

Anemia management and treatment for children in Mozambique are largely based on the WHO's Integrated Management of Childhood Illness (IMCl) protocols. These protocols call for the provision of a package of treatments for mild and moderate anemia, which includes iron supplementation, anti-helminth treatment, and oral antimalarials, if a malaria test is positive; or urgent referral to a hospital if the child has severe anemia. ${ }^{36}$ Although this syndromic diagnosis and management approach do rely on strong evidence as to the most common causes of

TABLE 4

Among HIV-infected children, the association of multiple concurrent hospital diagnoses with hemoglobin level

\begin{tabular}{lcc}
\hline \multicolumn{1}{c}{ Hospital diagnosis } & Adjusted contrast ${ }^{*}(95 \% \mathrm{Cl})$ & $P$-value \\
\hline $\begin{array}{l}\text { Bacteremia and malaria (vs. no } \\
\text { bacteremia and no malaria) }\end{array}$ & $-4.12(-5.85,-2.39)$ & $<.0001$ \\
$\begin{array}{l}\text { Bacteremia and malnutrition (vs. no } \\
\text { bacteremia and no malnutrition) }\end{array}$ & $-0.67(-1.65,0.30)$ & 0.156 \\
$\begin{array}{l}\text { Malaria and malnutrition (vs. no malaria } \\
\text { and no malnutrition) }\end{array}$ & $-1.28(-2.26,-0.29)$ & $<0.0001$ \\
\hline${ }^{*}$ Separate multiple linear regressions were used to assess the interaction of prespecified diagnosis and to estimate the contrasts of interest.
\end{tabular}


TABLE 5

Among HIV-infected children, the association of diagnosis with death during hospitalization using multiple logistic regression

\begin{tabular}{lcr}
\hline & Adjusted odds ratio* $(95 \% \mathrm{Cl})$ & $P$-value \\
\hline Anemia (per 1 g/dL decrease) & $1.02(0.87,1.20)$ & 0.767 \\
Bacteremia† & $3.97(1.61,9.78)$ & 0.003 \\
Malariat & $0.55(0.14,2.07)$ & 0.373 \\
Malnutrition & $1.88(0.80,4.43)$ & 0.147 \\
Acute respiratory illness & $1.18(0.29,2.06)$ & 0.009 \\
Gastroenteritis/diarrhea & $0.95(0.40,2.28)$ & 0.915 \\
\hline
\end{tabular}

* Adjusted odds ratio covariates were child gender, age, and health facility where hospitalized.

† Laboratory-confirmed diagnosis.

anemia in this age-group, its implementation is frequently applied without attempt to ascertain the true cause or contributors to one's anemia, nor trying to determine which component of the treatment package subsequently contributed to one's improvement.

Strategies for the control of anemia call for the implementation of mass prevention efforts that target, once again, "the most common causes of anemia." Over the last few decades, we have seen significant investments in efforts targeting anemia's largest contributors, such as campaigns for iron and multivitamin supplementation, deworming campaigns, and campaigns to prevent malaria, including bed net distribution and indoor residual spraying, among others. ${ }^{12}$ In many high HIV burden countries, such as Mozambique, these campaigns have been implemented simultaneously with large-scale increases in access to HIV care and treatment initiatives. ${ }^{37-39}$ Yet, despite all these, the global prevalence of anemia remains unacceptably high with only modest improvements over time. . $^{2,12}$

One reason for this may be that all too often, anemia is dealt with through a lens from which it is seen as a secondary consequence of many other processes, each that individually takes priority in control strategy debates. rather than anemia serving as the primary outcome metric from which multiple etiologies are attacked simultaneously for improvement. For example, Mozambique implements IMCl strategies for syndromic management of anemia; they implement broadscale malaria control programs, conduct frequent deworming and vitamin supplementation campaigns, and have regionally targeted acute and chronic malnutrition programs and large national programs for HIV care and treatment; all strategies recommended for the control of anemia. However, Mozambique lacks a harmonized strategy for coverage and uptake of each of these initiatives at the same time and place. This has resulted in incomplete or disjointed coverage, making it difficult to measure the impact of any individual or concurrent program being implemented.

Moreover, other less common yet still significant contributors to anemia are not being sufficiently addressed in Mozambique, as these are typically only included in small pilot programs or for research in a limited population. For example, in our hospitalized cohort, bacteremia contributed to lower $\mathrm{Hb}$ concentrations overall and a higher likelihood of death, yet the vast majority of Mozambique's population is not served by the handful of health facilities that have access to blood culture machines. We believe the limited scale of microbiology capacity in-country and its resulting undetected bacteremia to be an important contributor to the elevated prevalence of anemia in Mozambique and an important cause of preventable morbidity and mortality in Mozambican children with HIV. ${ }^{40}$ In addition, other studies in Mozambique have shown that although not common, disorders of $\mathrm{Hb}$ production do exist. For example, $\alpha$-thalassemia is present in about $50 \%$ of patients tested, potentially interfering with strategies to use $\mathrm{Hb}$ level as a marker of iron status and lessening justifications for iron supplementation as an anemia control measure. ${ }^{14,41,42}$

This study has several limitations. First, the study's primary objective was to determine the incidence, etiology, and other characteristics of bloodstream infections in febrile hospitalized children and, as such, was not designed specifically to identify the various etiologies of anemia in this cohort. We had age covariate missing data (4\%), and its missingness did not appear related to $\mathrm{Hb}$ values. Further sensitivity analysis conducted by applying $\mathrm{MI}$ methods had similar results (data not shown) when compared with the main analysis. This said, we cannot completely rule out the impact of age covariate missing or unmeasured confounders as with any observational study. The definitions and diagnostic testing used for determining one's HIV status are based on Mozambican national protocols; however, it must be recognized that some children older than 9 months with a positive HIV rapid test could represent an HIV-exposed uninfected child with continued presence of maternal HIV antibodies causing the test to be falsely positive. Nonetheless, we feel then likelihood of this to be very low, if any. Being a hospitalized study of HIV-infected and HIV-exposed uninfected children, our findings likely represent the severe end of the spectrum when it comes to anemic children and a higher proportion of concomitant morbidity and mortality. Future studies should be designed and adequately powered specifically to evaluate the effect of anemia on mortality. Finally, our findings cannot be generalized to the general population of children aged 6-59 months in Mozambique, and our regional focus on Zambézia and Maputo Provinces may limit generalizability to other regions of Mozambique as well.

\section{CONCLUSION}

In a hospitalized cohort of HIV-infected and HIV-exposed uninfected children aged 6-59 months, the prevalence of anemia was high, with most categorized as moderate anemia. The etiology of anemia in high burden malaria, HIV, TB, and poor nutrition countries, such as Mozambique, is multifactorial, and multiple etiologies may be contributing to one's anemia at any given time. It is important to involve the numerous health programs for malaria prevention and treatment, malnutrition, HIV care and treatment, TB control, and helminth control in a more harmonized approach. For example, health authorities could hold strategic planning meetings on childhood anemia, inviting representatives from each of the 
aforementioned sectors to map out the timing and coverage of interventions to be implemented so that they are integrated and leverage each other for maximum effect. Finally, clinical algorithms used by physician and nonphysician clinicians in Mozambique should incorporate integrated and non-diseasespecific approaches to pediatric anemia management and should include improved access to blood culture.

Received June 3, 2019. Accepted for publication December 13, 2019.

Published online January 13, 2020.

Acknowledgments: The authors are very grateful to the study subjects for their participation in this study. We would like to thank the laboratory staff of the Microbiology Department of the Faculty of Medicine at UEM as well as the MozBact study team at Central Hospital Maputo, Hospital Jose Macamo, General Hospital Mavalane, Central Hospital Quelimane, and General Hospital Quelimane.

Financial support: Research reported in this publication was supported by the National Institute of Allergy and Infectious Diseases of the National Institutes of Health under award number R01Al112295.

Disclaimer: The content is solely the responsibility of the authors and does not necessarily represent the official views of the National Institutes of Health.

Authors' addresses: Caitlyn Duffy, Department of Pediatrics, Vanderbilt University Medical Center, Nashville, TN, E-mail: caitlyn.t.duffy@vumc.org. Darlenne B. Kenga, Fabião E. Maússe, Alice Manjate, and Jahit Sacarlal, Department of Microbiology, Faculty of Medicine, University Eduardo Mondlane, Maputo, Mozambique, E-mails: darlene.bintikenga@gmail.com, fabiaomausse@ymail.com, alimanjate28@gmail.com, and jahityash2002@ gmail.com. Tebeb Gebretsadik, Department of Biostatistics, Vanderbilt University Medical Center, Nashville, TN, E-mail: tebeb.gebretsadik@ vumc.org. Emesto Zaqueu, Central Hospital Quelimane, Quelimane, Mozambique, E-mail: eflavio91@gmail.com. Hermenegilda F. Fernando, General Hospital Mavalane, Maputo, Mozambique, E-mail: hermefreanussa@gmail.com. Ann F. Green and Troy D. Moon, Vanderbilt Institute for Global Health, Vanderbilt University Medical Center, Nashville, TN, E-mails: ann.f.green@vumc.org and troy.moon@ vumc.org.

This is an open-access article distributed under the terms of the Creative Commons Attribution (CC-BY) License, which permits unrestricted use, distribution, and reproduction in any medium, provided the original author and source are credited.

\section{REFERENCES}

1. McLean E, Cogswell M, Egli I, Wojdyla D, de Benoist B, 2009. Worldwide prevalence of anaemia, WHO vitamin and mineral nutrition information system, 1993-2005. Public Health Nutr 12: 444-454.

2. Stevens GA, Finucane MM, De-Regil LM, Paciorek CJ, Flaxman SR, Branca F, Peña-Rosas JP, Bhutta ZA, Ezzati M; Nutrition Impact Model Study Group (Anaemia), 2013. Global, regional, and national trends in haemoglobin concentration and prevalence of total and severe anaemia in children and pregnant and non-pregnant women for 1995-2011: a systematic analysis of population-representative data. Lancet Glob Health 1: e16-e25.

3. Lopez A, Cacoub P, Macdougall IC, Peyrin-Biroulet L, 2016. Iron deficiency anaemia. Lancet 387: 907-916.

4. Magalhães RJS, Clements ACA, 2011. Mapping the risk of anaemia in preschool-age children: the contribution of malnutrition, malaria, and helminth infections in West Africa. PLOS Med 8: e1000438.

5. Enawgaw B, Workineh Y, Tadesse S, Mekuria E, Addisu A, Genetu $M, 2019$. Prevalence of anaemia and associated factors among hospitalized children attending the University of Gondar Hospital, northwest Ethiopia. EJIFCC 30: 35-47.

6. Grantham-McGregor S, Ani C, 2001. A review of studies on the effect of iron deficiency on cognitive development in children. J Nutr 131 (2 Suppl-2): 649S-666S.
7. Lawless JW, Latham MC, Stephenson LS, Kinoti SN, Pertet AM, 1994. Iron supplementation improves appetite and growth in anemic Kenyan primary school children. J Nutr 124: 645-654.

8. Oppenheimer SJ, 2001. Iron and its relation to immunity and infectious disease. J Nutr 131 (2 Suppl-2): 616S-633S.

9. Kiguli $S$ et al., 2015. Anaemia and blood transfusion in African children presenting to hospital with severe febrile illness. BMC Med 13: 21.

10. Schellenberg D, Schellenberg JR, Mushi A, Savigny DD, Mgalula L, Mbuya C, Victora CG, 2003. The silent burden of anaemia in Tanzanian children: a community-based study. Bull World Health Organ 81: 581-590.

11. Kassebaum NJ; GBD 2013 Anemia Collaborators, 2016. The global burden of anemia. Hematol Oncol Clin North Am 30: 247-308.

12. Joint Statement by the World Health Organization and the United Nations Children's Fund. Focusing on Anaemia, towards an Integrated Approach for Affective Anaemia Control. Available at: https://www.who.int/nutrition/publications/micronutrients/ WHOandUNICEF_statement_anaemia/en/. Accessed May 23, 2019.

13. Brentlinger PE, Silva WP, Vermund SH, Valverde E, Buene M, Moon TD, 2016. Practical management of HIV-associated anemia in resource-limited settings: prospective observational evaluation of a New Mozambican guideline. AIDS Res Hum Retroviruses 32: 12-25.

14. Crawley J, 2004. Reducing the burden of anemia in infants and young children in malaria-endemic countries of Africa: from evidence to action. Am J Trop Med Hyg 71 (Supp/ 2): 25-34.

15. Moraleda $\mathrm{C}$ et al., 2017. Anaemia in hospitalised preschool children from a rural area in Mozambique: a case control study in search for aetiological agents. BMC Pediatr 17: 63.

16. National Institute of Statistics, 2017. Preliminary Results IV, General Population Census 2017. Available at: http://www.ine.gov.mz/ operacoes-estatisticas/censos/censo-2007/censo-2017/resultadospreliminares-iv-rgph-2017/view. Accessed May 23, 2019.

17. Ministry of Health and the National Institute for Statistics, 2018. Mozambique Survey of Indicators of Immunization, Malaria, and HIVIAIDS (IMASIDA) 2015. Available at: https://dhsprogram.com/ pubs/pdf/AIS12/AIS12.pdf. Accessed May 23, 2019.

18. Mozambique Ministry of Health, 2017. National Program for the Control of STI and HIVIAIDS. Available at: http://www.misau.gov.mz/ index.php/its-hiv-sida. Accessed May 23, 2019.

19. García-Basteiro AL et al., 2016. Poor tuberculosis treatment outcomes in Southern Mozambique (2011-2012). BMC Infect Dis 16: 214.

20. Rose ES, Blevins M, González-Calvo L, Ndatimana E, Green AF, Lopez ML, Olupona O, Vermund SH, Moon TD, 2015. Determinants of undernutrition among children aged 6 to 59 months in rural Zambézia Province, Mozambique: results of two population-based serial cross-sectional surveys. BMC Nutr 1: 41.

21. Carlucci JG, Blevins Peratikos M, Cherry CB, Lopez ML, Green AF, González-Calvo L, Moon TD; Ogumaniha-SCIP Zambézia Consortium, 2017. Prevalence and determinants of malaria among children in Zambézia Province, Mozambique. Malar $J$ 16: 108.

22. Moon TD, Hayes CB, Blevins M, Lopez ML, Green AF, GonzálezCalvo L, Olupona O; Ogumaniha-SCIP Zambézia Consortium, 2016. Factors associated with the use of mosquito bed nets: results from two cross-sectional household surveys in Zambézia Province, Mozambique. Malar J 15: 196.

23. Fonseca AM, Fernandes N, Ferreira FS, Gomes J, Centeno-Lima S, 2014. Intestinal parasites in children hospitalized at the Central Hospital Maputo, Mozambique. J Infect Dev Ctries 8: 786-789.

24. Shari CR, Sawe HR, Murray BL, Mwafongo VG, Mfinanga JA, Runyon MS, 2017. Emergency blood transfusion practices among anaemic children presenting to an urban emergency department of a tertiary hospital in Tanzania. BMC Hematol 17: 19.

25. Woldie $\mathrm{H}$, Kebede $\mathrm{Y}$, Tariku A, 2015. Factors associated with anemia among children aged 6-23 months attending growth monitoring at Tsitsika Health Center, Wag-Himra zone, northeast Ethiopia. J Nutr Metab 2015: 928632. 
26. Harrel FE Jr., 2001. Regression Modeling Strategies. New York, NY: Springer-Verlag.

27. Redd AD, Avalos A, Phiri K, Essex M, 2007. Effects of HIV type 1 infection on hematopoiesis in Botswana. AIDS Res Hum Retroviruses 23: 996-1003.

28. Gedefaw L, Yemane T, Sahlemariam Z, Yilma D, 2013. Anemia and risk factors in HAART naïve and HAART experienced HIV positive persons in south west Ethiopia: a comparative study. PLoS One 8: e72202.

29. Prendergast A, Walker AS, Mulenga V, Chintu C, Gibb DM, 2011. Improved growth and anemia in HIV-infected African children taking cotrimoxazole prophylaxis. Clin Infect Dis 52: 953-956.

30. Dryden-Peterson S et al., 2013. Cotrimoxazole prophylaxis and risk of severe anemia or severe neutropenia in HAARTexposed, HIV-uninfected infants. PLoS One 8: e74171.

31. Dow A et al., 2012. The effects of cotrimoxazole prophylactic treatment on adverse health outcomes among human immunodeficiency virus-exposed, uninfected infants. Pediatr Infect Dis J 31: 842-847.

32. Ivan E, Crowther NJ, Rucogoza AT, Osuwat LO, Munyazesa E, Mutimura E, Njunwa KJ, Zambezi KJB, Grobusch MP, 2012. Malaria and helminthic co-infection among HIV-positive pregnant women: prevalence and effects of antiretroviral therapy. Acta Trop 124: 179-184.

33. Fincham JE, Markus MB, Adams VJ, 2003. Could control of soiltransmitted helminthic infection influence the HIV/AIDS pandemic. Acta Trop 86: 315-333.

34. Kupka R, Msamanga GI, Mugusi F, Petraro P, Hunter DJ, Fawzi WW, 2007. Iron status is an important cause of anemia in HIVinfected Tanzanian women but is not related to accelerated HIV disease progression. J Nutr 137: 2317-2323.
35. Nyesigire Ruhinda E, Bajunirwe F, Kiwanuka J, 2012. Anaemia in HIV-infected children: severity, types and effect on response to HAART. BMC Pediatr 12: 170.

36. World Health Organization, 2014. Integrated Management of Childhood Illness, Distance Learning Course, Module 6, Malnutrition and Anaemia. Available at: https://apps.who.int/iris/ bitstream/handle/10665/104772/9789241506823_Module6 eng.pdf;jsessionid=F0C9340AB4A83DC821C5189945821770? sequence $=8$. Accessed May 23, 2019.

37. Vermund SH, Blevins M, Moon TD, José E, Moiane L, Tique JA, Sidat M, Ciampa PJ, Shepherd BE, Vaz LME, 2014. Poor clinical outcomes for HIV infected children on antiretroviral therapy in rural Mozambique: Need for program quality improvement and community engagement. PLoS One 9: e110116.

38. Ciampa PJ, Tique J, Jumá N, Sidat M, Moon TD, Rothman RL, Vermund SH, 2012. Addressing poor retention of infants exposed to HIV: a quality improvement study in rural Mozambique. J Acquir Immune Defic Syndr 60: e46-e52.

39. Moon TD et al., 2010. Lessons learned while implementing an HIV/AIDS care and treatment program in rural Mozambique. Retrovirol Res Treat 3: 1-14.

40. Moon TD, Silva WP, Buene M, Morais L, Valverde E, Vermund SH, Brentlinger PE, 2013. Bacteremia as a cause of fever in ambulatory, HIV-infected Mozambican adults: results and policy implications from a prospective observational study. PLoS One 8: e83591.

41. Willcox MC, Liljestrand J, Bergström S, 1986. Abnormal haemoglobins among pregnant women from Mozambique. $J$ Med Genet 23: 151-152.

42. Aguilar R, Moraleda C, Quintó L, Renom M, Mussacate L, Macete E, Aguilar JL, Alonso PL, Menéndez C, 2012. Challenges in the diagnosis of iron deficiency in children exposed to high prevalence of infections. PLoS One 7: e50584. 